Received: 2011.08 .05 Accepted: 2011.09 .02

\title{
Congenital chylous ascites
}

\section{Justyna Romańska-Kita ${ }^{1}$, Maria Katarzyna Borszewska-Kornacka ${ }^{1}$, Anna Dobrzańska², Iwona Rudzińska1 ${ }^{1}$ Justyna Czech-Kowalska², Tomasz Wawrzoniak ${ }^{1}$}

\author{
${ }^{1}$ Clinic of Neonatology and Neonate Intensive Care, Medical University of Warsaw, Warsaw, Poland \\ ${ }^{2}$ Clinic of Neonatology, Neonate Pathology and Intensive Care, Children's Memorial Health Institute, Warsaw, Poland
}

Author's address: Maria Katarzyna Borszewska-Kornacka, Clinic of Neonatology and Neonate Intensive Care, Medical University of Warsaw, Karowa 2 Str., 00-315 Warsaw, Poland, e-mail: mariak@szpitalkarowa.p

Key words: $\quad$ newborn • congenital chylous ascites • paracentesis somatostatin-octreotide

PDF file: $\quad$ http://www.polradiol.com/fulltxt.php?ICID $=881954$

\section{Background}

Congenital abdominal chylous ascites in newborns is a rare entity, conditioned by numerous factors and with changing dynamics of the disease. Because of the lack of therapeutic and diagnostic standards, this disease constitutes to be a medical challenge and individual therapy seems to be extremely important. This article presents current knowledge on pathogenesis, diagnostics and management of this disease, as well as a case report of a newborn with primary congenital chylous ascites in the abdominal cavity.

\section{Pathogenesis}

The aetiological factors of abdominal chylous ascites in the paediatric population can be primary or secondary. Abnormalities connected with the development and maturation of lymphatic vessels are the cause of the primary process (a much more common one). Dysplastic lymphatic vessels, both hypo- and hyperplastic ones [widening of lymphatic vessels, lymphangiomas] favour lymphatic leakage in the mechanism of increasing pressure in the lymphatic system and lymphatic congestion [1-3]. Delayed maturation of lymphatic vessels, leading to abnormal structure of their walls, also results in lymphatic leakage to the peritoneal cavity. Developmental disorders of lymphatic vessels constitute the main cause of congenital abdominal chylous ascites found in newborns. The presence of these anomalies in genetic syndromes - Turner syndrome, yellow nail syndrome, Klippel-Trenaunay-Weber syndrome and in twins, as well as children of related parents, points to the role of genetic factors in etiopahtogenesis of disorders of lymph circulation $[4,5]$. Lymph accumulation in the peritoneal cavity is less frequent in the course of neoplastic processes, specific and nonspecific inflammatory processes, parasitic infections, enlarged mesenteric lymph nodes, after radiotherapy, and due to injuries of lymphatic vessels. Apart from iatrogenic injuries that are usually a consequence of surgeries with opening of the abdominal cavity, there are also non-accidental injuries (abusechild syndrome), with newborns and young children being at particular risk $[6,7]$.

\section{Diagnostics}

Symptoms of abdominal chylous ascites in newborns follow from the presence of the fluid in the peritoneal cavity, increased pressure in the abdominal cavity, and abnormal function of the intestines. They usually include: vomiting, loss of appetite, increased abdominal circumference, presence of inguinal hernias, scrotal and vaginal oedema, and symptoms suggestive of the presence of free fluid in the abdominal cavity. With an increasing volume of lymph in the peritoneal cavity, symptoms of respiratory failure arise. Abnormal function of the GI tract results in malabsorption of nutrients in the intestines [6]. 
A key stage in the diagnostics of abdominal chylous ascites is paracentesis $[4,8]$. Ultrasound and radiological examination of the abdominal cavity are able to confirm the presence of free fluid in the peritoneal cavity, without determining its origin. Nevertheless, they are usually applied in the diagnostic process, for excluding the presence of a mass lesion in the abdominal cavity, and allowing for a safe paracentesis. Due to their noninvasiveness, they may be repeated and thus it is possible to evaluate the course of the pathological process. In the macroscopic evaluation, lymph is a cloudy milky or straw-coloured fluid (the colour depends on the diet). A typical feature of the lymph is a high concentration of triglycerides [over $1000 \mathrm{mg} / \mathrm{dl}$, significantly exceeding the levels found in the serum. The presence of chylomicrons is considered to be pathognomic $[2,4]$. Next, the diagnostic process consists in determining the causes of abnormal lymph circulation.

Lymphangiography still remains the 'golden standard' in visualisation of the lymphatic system, although it is technically difficult to carry out and connected with a high risk in newborns and infants [4]. It seems that radiological examinations are replaced by radioisotope techniques. Lymphoscintigraphy is a method showing the site of lymph leakage, connected with a lower risk of complications, and offering a possibility of repeating the evaluation [2]. Invasive techniques - laparoscopy and laparotomy, still remain a diagnostic and therapeutic tool used in abdominal chylous ascites resistant to conservative treatment. Visualisation of the site of lymph leakage by providing the patient with a high-fat meal directly before the procedure, together with pre- or intraoperative administration of fatsoluble dyes seems to be of the highest importance for the success of these methods $[2,4]$.

\section{Treatment}

Treatment of abdominal chylous ascites is a multidirectional process. It consists of a few therapeutic stages, starting with the least invasive and tiring one (for the patient), to surgical procedures treated usually as a last resort. An important part includes activities aimed at optimisation of nutritional habits in order to avoid nutritional deficiencies being characteristic for the disease and constituting a complication of the therapy [9]. Diet based on specialist formulas rich in MCTs is a popular conservative method of treatment, considered as a 'first-line' intervention. Its disadvantages include: a long time of use, i.e. from 4 to 6 months, necessary for the therapeutic effect, as well as estimated success rate of about 30\% [10]. A next stage is the withdrawal of enteral nutrition, with introduction of parenteral nutrition. No food supply results in a decrease in lymph production, enabling the process of reconstruction of the lymphatic vessels, with reduction of the risk of nutritional deficits. According to Bhatia et al., a satisfactory result can be obtained after 3-4 weeks of treatment. The rate of success reaches $60-100 \%$. However, it is connected with a risk accompanying the long-term use of parenteral nutrition [4]. The first information on a successful use of somatostatin in the treatment of lymphatic system disorders appeared in the literature in 1990 and concerned chylothorax in an adult person [11]. Further studies presented new, enthusiastic data on the use of somatostatin and its synthetic analogue - octreotide, in the treatment of diseases accompanied by lymph accumulation in the pleural cavities and peritoneal cavity, in adults, newborns, infants, and children $[12,13]$. Due to a very rare incidence of this disorder in children, the literature includes mainly case reports. Positive results, including the reduction and regression of chylous ascites, were observed as early as after a few days following treatment introduction. According to Huang et al., there were four case reports of patients with congenital abdominal chylous ascites treated with somatostatin, published by the year 2011 [14]. A probable mechanism of action includes a decrease in lymph production and its flow rate. Somatostatin with parenteral nutrition and withheld enteral nutrition or with enteral nutrition based on a diet including MCTs, shows a similar rate of success, although there are no data from randomised trials which would be able to confirm this. Possible adverse effects of the therapy include disorders of carbohydrate metabolism, nausea, diarrhea, abdominal distension, and abnormal liver function. More alarming for neonatologists is the information on a potential relationship between octreotide and necrotising enterocolitis, as well as induced pulmonary hypertension, especially in the context of a growing problem of premature neonates, with congenital disorder [14,15]. An increasing popularity of somatostatin follows from the ease in its administration, its low invasiveness, short treatment time, and its high effectiveness [14].

Surgery is advised in cases resistant to conservative treatment, which is usually undertaken for $4-8$ weeks. Before a planned surgical intervention, CT, MRI and laparoscopic methods are applied to define the type of lymphatic vessel pathology and its location [2]. The use of fibrin glue, especially in the situation of multiple lymph leakage sites, seems to be a successful method supporting the classic surgical techniques $[2,4,16,17]$. A shunt joining the peritoneal cavity with the venous system is treated as a last resort, due to the possibility of severe complications, and a limited effectiveness $[1,2,4]$. In a review of case reports of abdominal chylous ascites in newborns and children, carried out by Cochran et al., surgical treatment was found to be successful in $44 \%$ of cases. In the following years, with a simultaneous development of modern surgical techniques, and especially microsurgeries and laparoscopic surgeries, the recovery rate reached $80-100 \%$ [9].

\section{Case Report}

A full-term female neonate, born at 38 weeks by spontaneous delivery, in a good general health state, with birth weigh of $3.2 \mathrm{~kg}$. The course of pregnancy complicated by pregestational diabetes of class A, hypothyroidism, and abdominal ascites of the foetus diagnosed in 37th hbd. A physical examination carried out after the delivery revealed a distended and rigid abdomen. No dysmorphic features were found. After introduction of enteral nutrition, the abdominal circumference started to increase gradually, accompanied by increasing respiratory failure tachypnoe, with activation of additional respiratory muscles. Due to mild respiratory disturbances, the baby did not require support or mechanical ventilation. Its karyotype was considered normal. Infection with cytomegalovirus or T. gondii, as well as metabolic disorders were excluded 


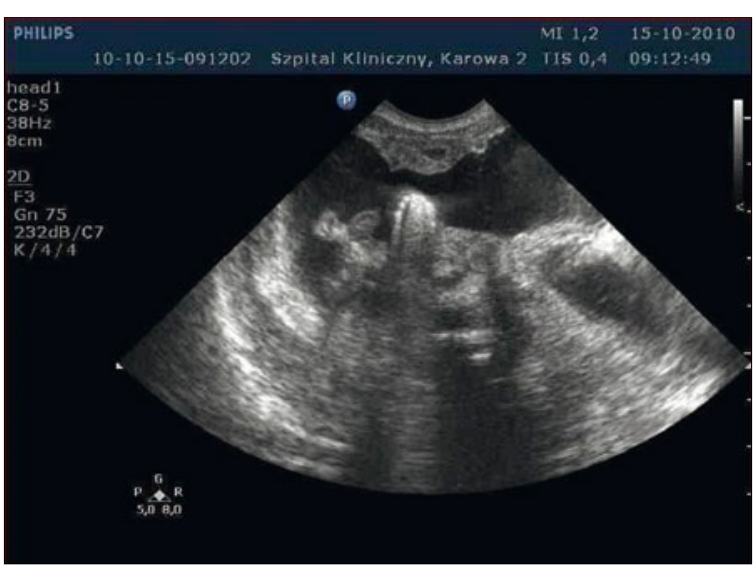

Figure 1. Congenital chylous ascites.

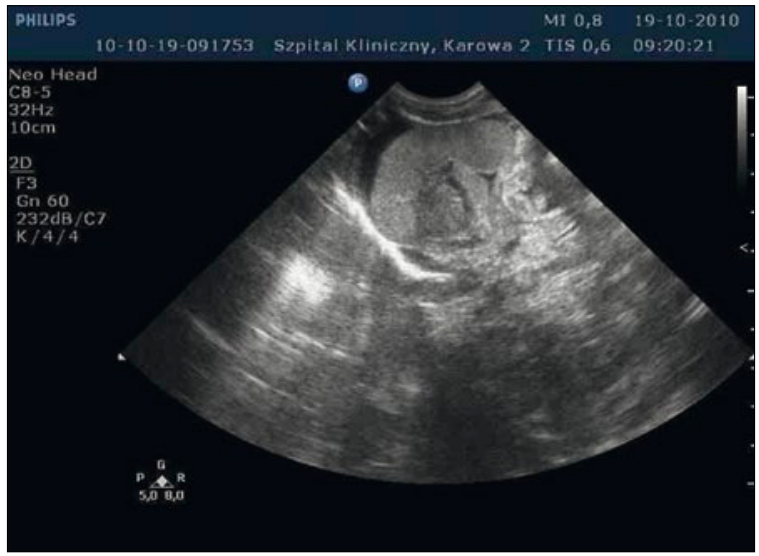

Figure 2. Congenital chylous ascites after paracentesis.

with MS/MS and GC/MS tests. Echocardiography showed a patent foramen ovale and slightly enlarged right atrium and right ventricle. Ultrasound examinations of abdominal organs and brain did not reveal congenital malformations. Ultrasound examination of the abdominal cavity showed fluid in the parietal cavity and normal chest organs (Figure 1). On day 4, paracentesis was performed, with obtaining $50 \mathrm{ml}$ of a yellow, cloudy fluid (Figure 2). The analysis of the peritoneal fluid showed an increased level of triglycerides, typical for the lymph, substantially exceeding the concentrations for the serum. Due to increasing abdominal ascites, the procedure was repeated on day 7 of life. A volume of $150 \mathrm{ml}$ of a cloudy, amber-coloured fluid was obtained. From day 4 to day 10, enteral nutrition was withheld. Parenteral nutrition was started and continued until the end of the second week of life. No fat emulsions were used for nutritional support. From day 9 to day 25 , octreotide was administered by continuous infusion, at a rate of $1 \mathrm{mcg} / \mathrm{kg} / \mathrm{h}$ (Figure 3). On day 9, a permanent drain was introduced into the peritoneal cavity. On the following days, a substantial reduction in the amount of drained lymph was observed. The drain was removed after 7 days (Figure 3). Enteral nutrition with formulas including MCTs, and then natural food, was started on day 10 of life, with gradually increased amounts. From the $4^{\text {th }}$ week of life, the baby was fed with mother's milk only. At follow-up visits, the health state of the baby remained good. No recurrence of lymph accumulation in the peritoneal cavity was found.

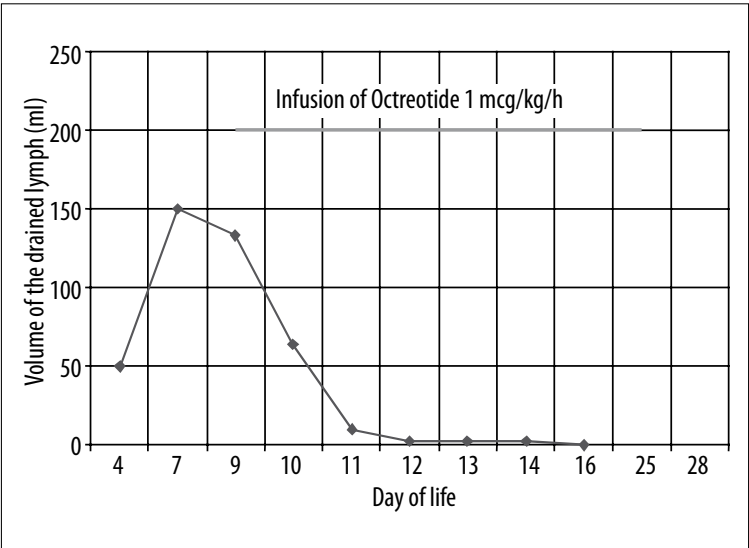

Figure 3. Reduction of chylous ascites after treatment.

\section{Discussion}

Congenital chylous ascites is a disease with a severe prognosis. With advancements in medicine, the mortality decreased substantially, i.e. from $27-30 \%$ to $12-17 \%$ $[9,18-20]$. Diagnostics and treatment of abdominal chylous ascites remains a controversial issue. Due to multifactorial etiopathogenesis, the diagnostic process should be individualised. As there are no standards of treatment, it is necessary to aim at treatment intensification [18]. Doubts connected with the diagnostic process concern the role of invasive and advanced diagnostic techniques, such as lymphangiography, laparoscopy, magnetic resonance imaging, and computed tomography. No advantages of MR and CT imaging were found over abdominal ultrasound examination, which is a widely available and safe examination. Lymphangiography, lymphoscintigraphy, laparoscopy, and diagnostic laparotomy are specialist methods available only in selected centers (methods of limited availability). Due to the possibility of successful conservative treatment of congenital abdominal chylous ascites, diagnostics for identification of the type and location of the lymphatic system malformations seems to be justified in case of resistance to this type of treatment and planning of surgery. Karagol et al. developed their own treatment scheme, according to which the therapy should begin with a few weeks of enteral nutrition with a diet including fat emulsion in the form of MCT. Next, enteral nutrition should be withheld, and parenteral nutrition should be started, with administration of somatostatin. The last stage is the surgery [4]. Treatment by diet modifications is connected with many disadvantages. It needs a few weeks, and thus a long-term hospitalisation. Simultaneous procedures of paracentesis as well as keeping the drain in the peritoneal cavity for weeks, are connected with a risk of complications. We should keep in mind the risk of deficiency in lymphatic components (due to an irreversible loss of the lymph). Moreover, attempts at transfusing lymph drained from the peritoneal cavity belong to the past $[4,18]$. Should octreotide, a safe method considerably shortening the duration of therapy, become the cornerstone of conservative treatment? Based on enthusiastic reports, it seems that it should. A more aggressive and invasive method including parenteral nutrition with simultaneous administration of somatostatin/octreotide, as a first-line treatment, seems to be more advantageous as concerns shorter time of treatment and 
hospitalization, and lower treatment costs. A low incidence of the pathology is the cause of the lack of randomised, prospective studies which would show an advantage of one form of treatment over another. One more controversy is the time for which the attempts of conservative and symptomatic treatment should be undertaken. The majority of the available reports published in the scientific literature concerning chylous ascites mention a period of 4-8 weeks, as the most appropriate, before making the decision on surgery [22]. In a report of 103 paediatric patients with chylous ascites, there were $63.9 \%$ of recoveries after conservative treatment, with a mean time of therapy amounting to 63.5 days [18,21]. Conservative treatment reducing chylous ascites, is of fundamental importance in stabilising patient's condition and preparing him/her to surgery. When assessing the odds of therapeutic success, it is important to determine the causes of chylous ascites [18]. Primary chylous ascites, being usually an idiopathic disorder, is connected with a much better prognosis, while the therapeutic success in the secondary chylous ascites depends on the severity of the main disease.

\section{Conclusions}

Congenital chylous ascites is a rare disease in newborns. No standards of care for newborns with chylous ascites complicates the diagnostic and therapeutic process. It is especially important to intensify and individualise the treatment process. Conservative treatment, with an early introduced administration of somatostatin seems to be advantageous, as it shortens the time of treatment and hospitalisation. Surgery should be considered after ineffective attempts at conservative treatment continued for 4-8 weeks. Before surgery, it seems necessary to try to establish the type and location of the pathology of lymphatic vessels, with the use of noninvasive and invasive diagnostic methods.

\section{References:}

1.te Pas AB, vd Ven K, Stokkel MPM et al: Intractable congenital chylous ascites. Acta Paediatr, 2004; 93: 1403-5

2. Campisi C, Bellini C, Eretta C et al: Diagnosis and management of primary chylous ascites. J Vasc Surg, 2006;43: 1244-48

3. Servelle M: Congenital malformation of the lymphatics of the small intestine. J Cardiovasc Surg, 1991; 32: 159-65

4. Karagol B, Zenciroglu A, Gokce S et al: Therapeutic management of neonatal chylous ascites: report of a case and review of the literature. Acta Paediatr, 2010; 99: 1307-10

5. Aalami O, Allen D, Organ C: Chylous ascites: a collective review. Surgery, 2000; 128: 761-78

6. Beshay V, Beshay J, Rosenberg A: Chylous ascites: a case of child abuse and an overview of a rare condition. J Pediatr Gastroenterol Nutr, 2001; 32: 487-89

7. Olazagasti JC, Fitzgerald JF, White SJ et al: Chylous ascites: a sign of unsuspected child abuse. Pediatrics, 1994; 94: 737-39

8. Guzoglu N, Erdeve O, Yilmaz Y et al: Intraperitoneal extravasation from umbilical venous catheter in differential diagnosis of neonatal chylous ascites. Acta Paediatr, 2010; 99: 1284

9. Cochran W, Klish W, Brown M et al: Chylous ascites in infants and children: a case report and literature review. J Pediatr Gastroenterol Nutr, 1985; 4: 668-73

10. Bhatia C, Pratap U, Slavik Z: Octreotide therapy: a new horizon in treatment of iatrogenic chyloperitoneum. Arch Dis Child, 2001; 85: 234-35

11. Ulibarri JI, Sanz Y, Fuentes C et al: Reduction of lymphorrhagia from ruptured thoracic duct by somatostatin. Lancet, 1990; 336: 258
12. Hwang JB, Choi SO, Park WH: Resolution of refractory chylous ascites after Kasai portoenterostomy using octreotide. J Pediatr Surg, 2004; 39: 1806-7

13. Au M, Weber T, Fleming R: Successful use of somatostatin in a case of neonatal chylothorax. J Pediatr Surg, 2003;38: 1106-7

14. Huang Y, Zhuang S, Li Y et al: Successful management of congenital chylous ascites in a premature infant using somatostatin analogue. Indian J Pediatr, 2011;78: 345-47

15. Arevalo RP, Bullabh P, Krauss AN et al: Octreotide-induced hypoxemia and pulmonary hypertension in premature neonates. $\mathrm{J}$ Pediatr Surg, 2003;38: 25l-53

16. Melo-Filho AA, Souza IJN, Leite CAC et al: Refractory congenital chylous ascites. Indian J Pediatr, 2010; 77(11): 1335-37

17. Zeidan S, Delarue A, Rome A et al: Fibrin glue application in the management of refractory chylous ascites in children. J Pediatr Gastroenetrol Nutr, 2008; 46: 478-81

18. Huang Y, Xu H: Successful treatment of neonatal idiopathic chylous ascites with total parenteral nutrition and somatostatin. HK J Paediatr, 2008;13: 130-34

19. Gribetz D, Kanof A: Chylous ascites in infancy; with a report of a case with vitamin A absorption study. Pediatrics, 1951; 7: 632

20. Bhatia C, Pratap U, Slavik Z: Octreotide therapy: a new horizon in treatment of iatrogenic chylipoeritoneum. Arch Dis Child, 2001; 85: 234-35

21. Mitsunaga T, Yoshida H, Iwai J et al: Successful surgical treatment of two cases of congenital chylous ascites. J Pediatr Surg, 2001; 36: 1717-19

22. Unger SW, Chandler JG: Chylous ascites in infants and children. Surgery, 1983; 93: 455-61 